\title{
Effects of Exercise Interventions on Physical, Social, and Sexual Function of Men with Prostate Cancer: A Meta-Analysis
}

\author{
${ }^{1}$ Yuan-Yuan Fang, RN, PhC, ${ }^{1}$ Yun-Hsiang Lee, PhD, RN; ${ }^{2}$ Jui-Chun Chan, MSN, RN; \\ ${ }^{3}$ Wen-Tzu Chiu, MSN, RN; ${ }^{1}$ Xiao-Yin Chou, PhC, RN; ${ }^{2}$ Chia-Tai Hung, PhD, RN* \\ ${ }^{1}$ School of Nursing, College of Medicine, National Taiwan University, Taipei, Taiwan. \\ 2 Department of Nursing, Mackay Medical College, New Taipei City, Taiwan. \\ Department of Nursing, National Taiwan University Hospital, Taipei, Taiwan.
}

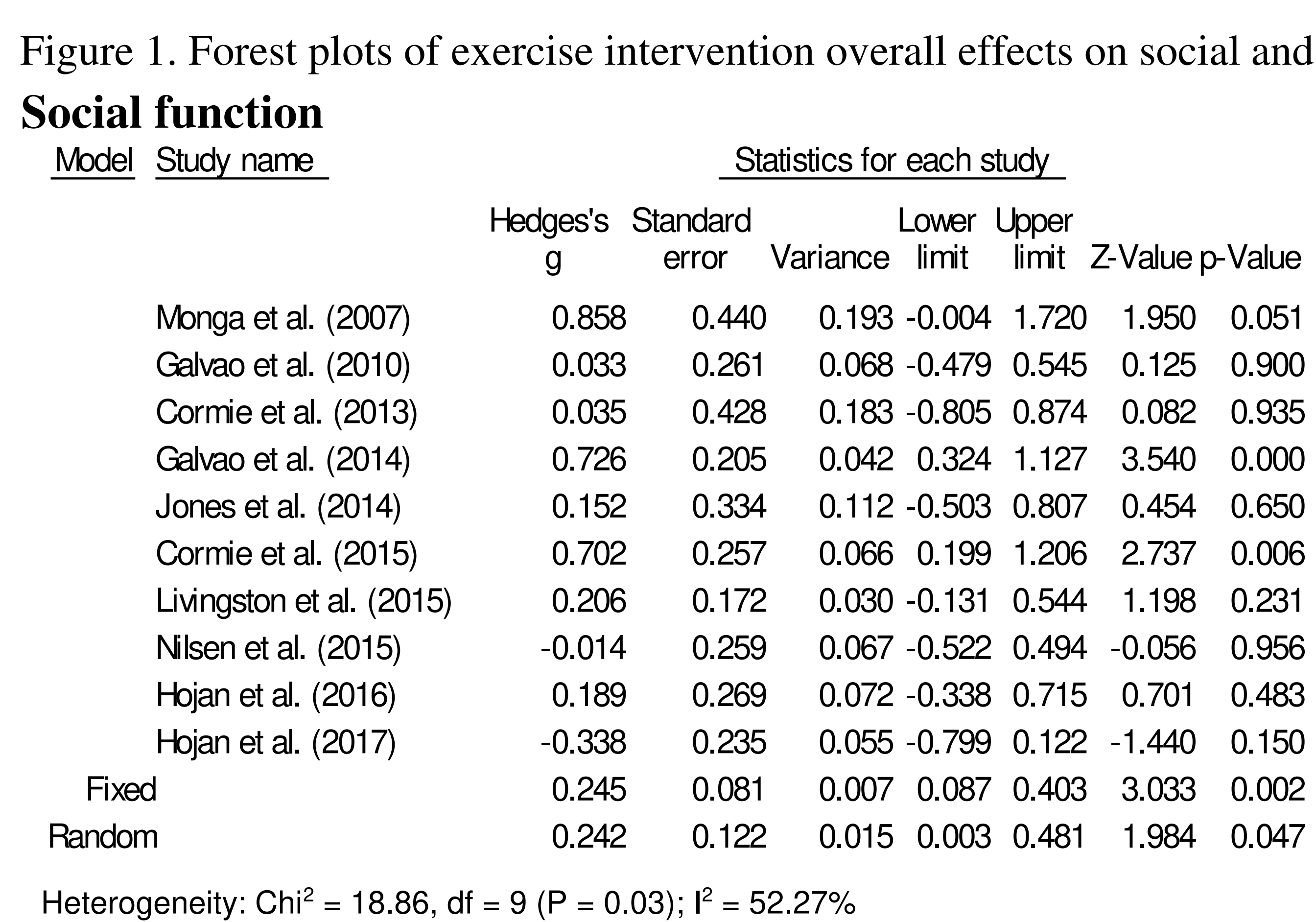

Physical function

Model Study name

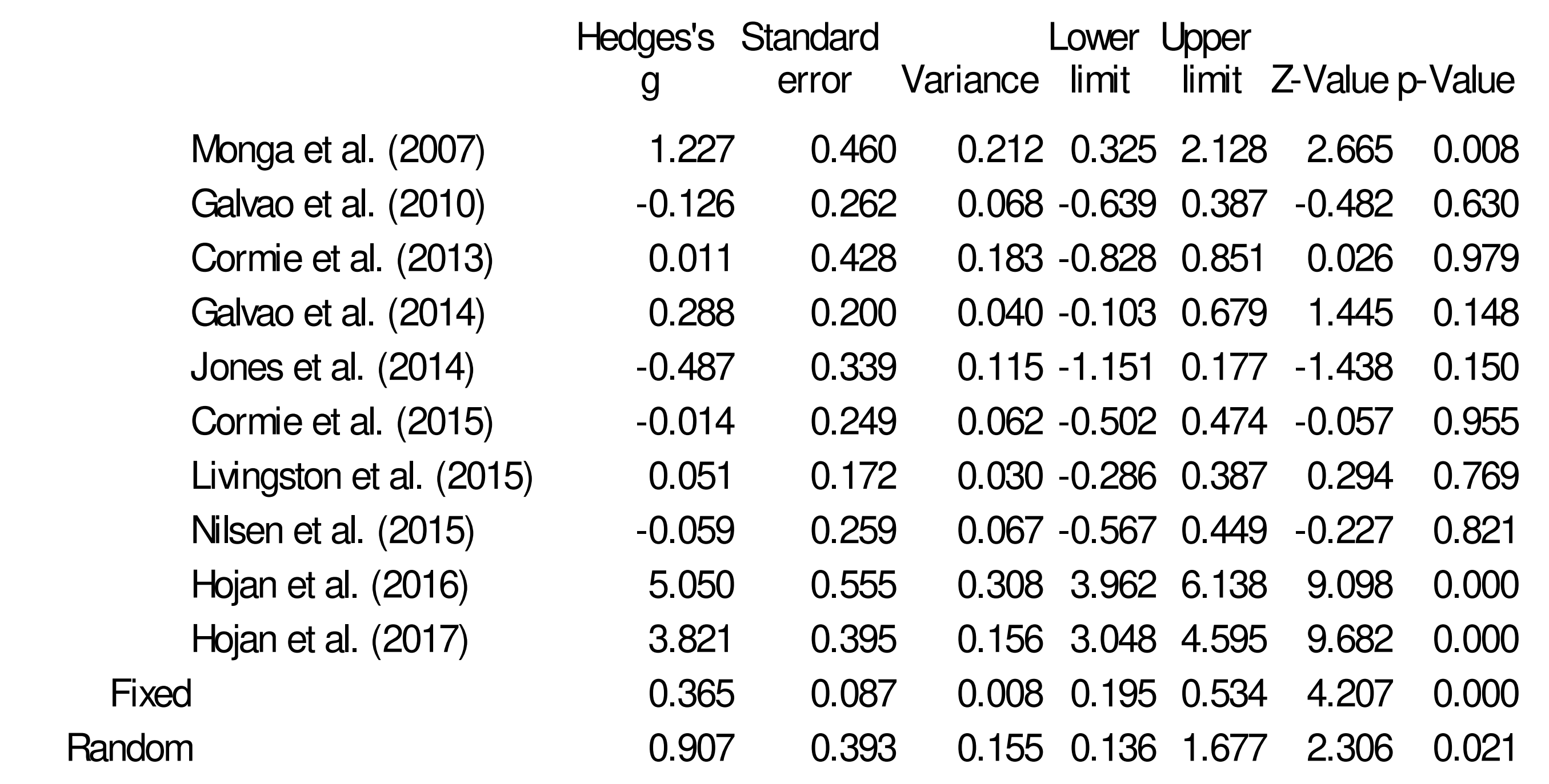

Heterogeneity: Chi $^{2}=170.46, \mathrm{df}=9(\mathrm{P}<0.01) ; \mathrm{l}^{2}=94.72 \%$

Mental Function

Model Study name

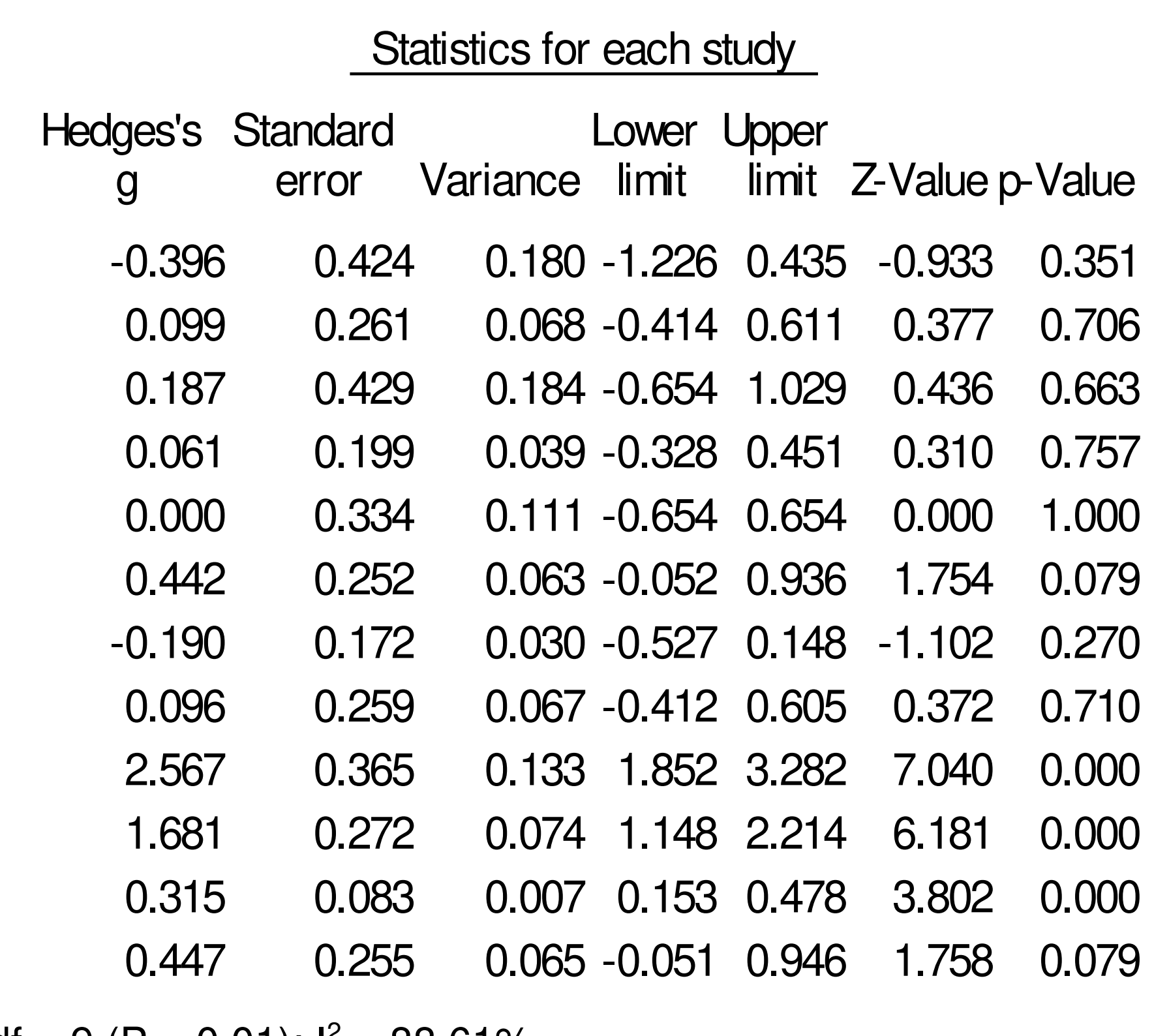

Random

:

Sexual activity

Model Study name

\begin{tabular}{|c|c|c|c|c|c|c|}
\hline \multicolumn{7}{|c|}{ Statistics for each study } \\
\hline $\begin{array}{l}\text { Hedges's } \\
\text { g }\end{array}$ & $\begin{array}{l}\text { Standard } \\
\text { error }\end{array}$ & Variance & $\begin{array}{l}\text { Lower L } \\
\text { limit }\end{array}$ & $\begin{array}{c}\text { Upper } \\
\text { limit }\end{array}$ & z-Value & p-Value \\
\hline 0.301 & 0.250 & 0.063 & $3-0.190$ & 0.792 & 21.202 & 0.229 \\
\hline 0.469 & 0.272 & 0.074 & $4-0.064$ & 1.003 & $\begin{array}{ll}3 & 1.726\end{array}$ & 0.084 \\
\hline 0.558 & 0.238 & 0.057 & 70.092 & 1.024 & $\begin{array}{ll}4 & 2.348\end{array}$ & 0.019 \\
\hline 0.446 & 0.146 & 0.021 & 0.160 & 0.731 & 13.061 & 0.002 \\
\hline 0.446 & 0.146 & 0.021 & 0.160 & 0.731 & 13.061 & 0.002 \\
\hline
\end{tabular}

Random

Cormie et al. (2015)

Hojan et al. (2016)

Hojan et al. (2017)

Cormie et al. (2013)

(2014)

(2015)

(2016)

Hojan et al. (2017)

$\begin{array}{lllllll}0.446 & 0.146 & 0.021 & 0.160 & 0.731 & 3.061 & 0.002\end{array}$

Heterogeneity: $\mathrm{Chi}^{2}=0.57, \mathrm{df}=2(\mathrm{P}=0.75) ; \mathrm{I}^{2}<0.00 \%$

Sexual function

Model Study name

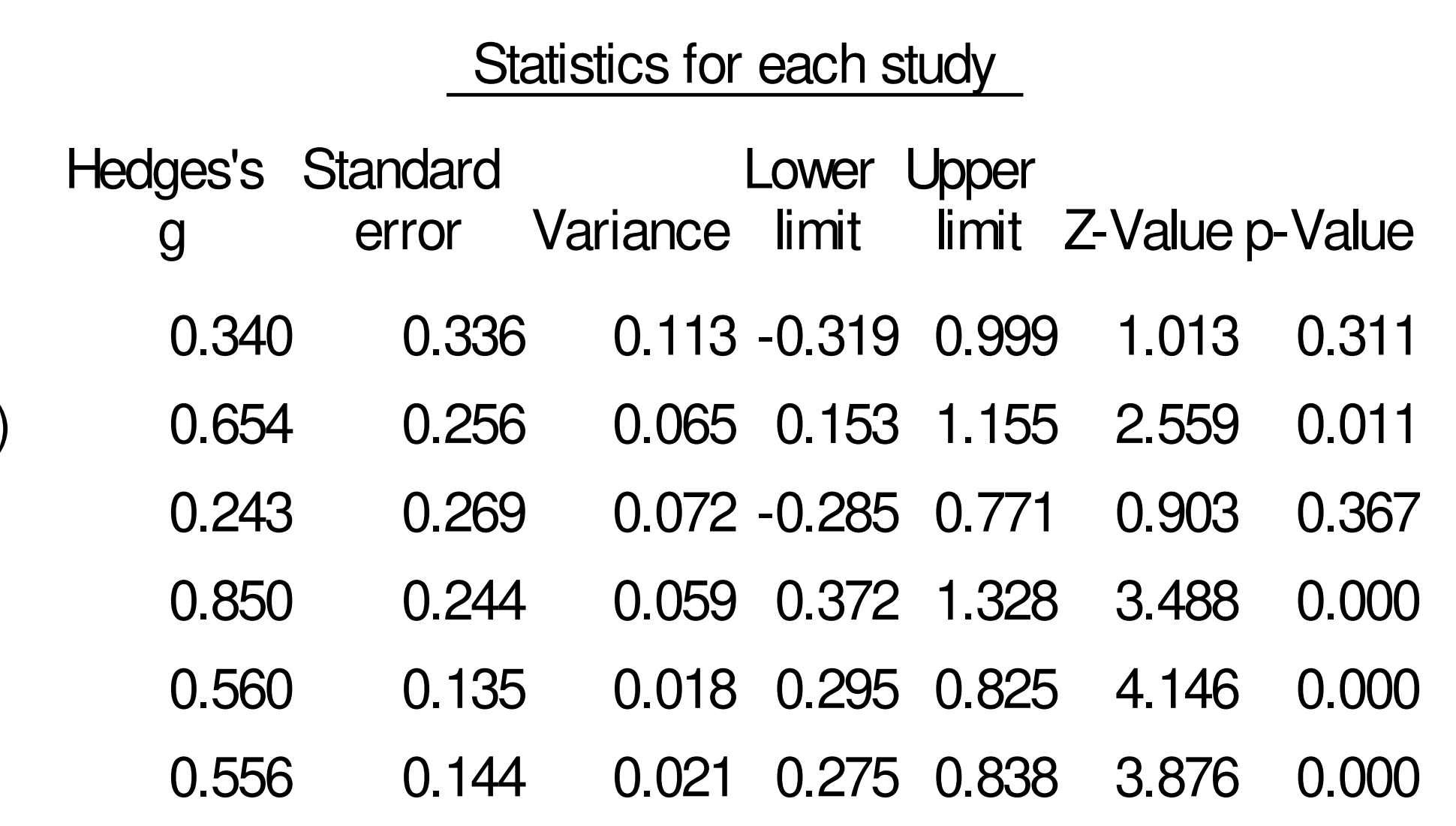

Random

Jone et al. (2014)

Cormie et al. (2015)

Hojan et al. (2016)

Hojan et al. (2017)

$(P=0.34) ; I^{2}=10.79 \%$

Depression

Model Study name

$$
\begin{aligned}
& \text { Std diff Standard Lotatistics for each study } \\
& \begin{array}{l}
\text { Std diff Standard } \\
\text { in means error Variance limit limit } Z \text {-Value p-Value }
\end{array} \\
& \begin{array}{lllllll}
0.209 & 0.173 & 0.030 & -0.130 & 0.549 & 1.209 & 0.227 \\
0.203 & 0.122 & 0.015 & -0.035 & 0.441 & 1.671 & 0.095
\end{array} \\
& \begin{array}{lllllll}
0.203 & 0.122 & 0.015 & -0.035 & 0.441 & 1.671 & 0.095
\end{array}
\end{aligned}
$$$$
\begin{array}{llllllll}
\text { Monga et al. (2007) } & 0.281 & 0.439 & 0.193 & -0.580 & 1.142 & 0.640 & 0.522
\end{array}
$$$$
\begin{array}{llllllll}
\text { Cormie et al. (2013) } \quad 0.254 & 0.449 & 0.202 & -0.626 & 1.134 & 0.566 & 0.571
\end{array}
$$$$
\begin{array}{llllllll}
\text { Jones et al. (2014) } & 0.000 & 0.342 & 0.117 & -0.669 & 0.669 & 0.000 & 1.000
\end{array}
$$$$
\begin{array}{llllllll}
\text { Cormie et al. (2015) } & 0.259 & 0.253 & 0.064 & -0.237 & 0.755 & 1.024 & 0.306
\end{array}
$$$$
\begin{array}{llllllll}
\text { Livingston et al. (2015) } & 0.209 & 0.173 & 0.030 & -0.130 & 0.549 & 1.209 & 0.227
\end{array}
$$$$
\text { Fixed }
$$

\section{Hedges's $\mathrm{g}$ and $95 \% \mathrm{Cl}$}

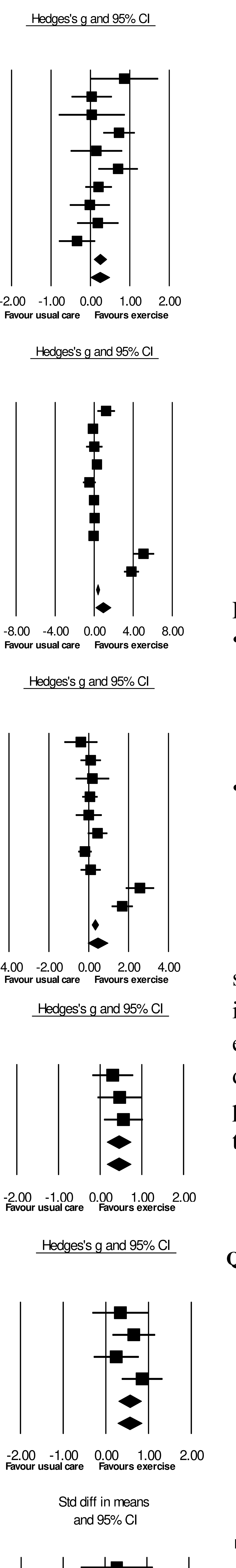

Hedges's $\mathrm{g}$ and $95 \% \mathrm{Cl}$

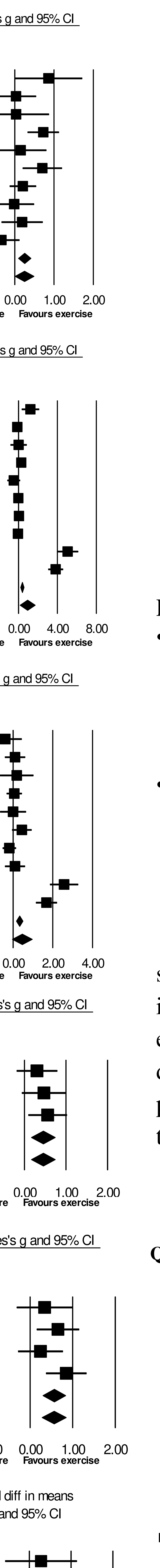

$\begin{array}{lllll}-8.00 & -4.00 & 0.00 & 4.00 & 8.00\end{array}$

Hedges's $\mathrm{g}$ and $95 \% \mathrm{Cl}$

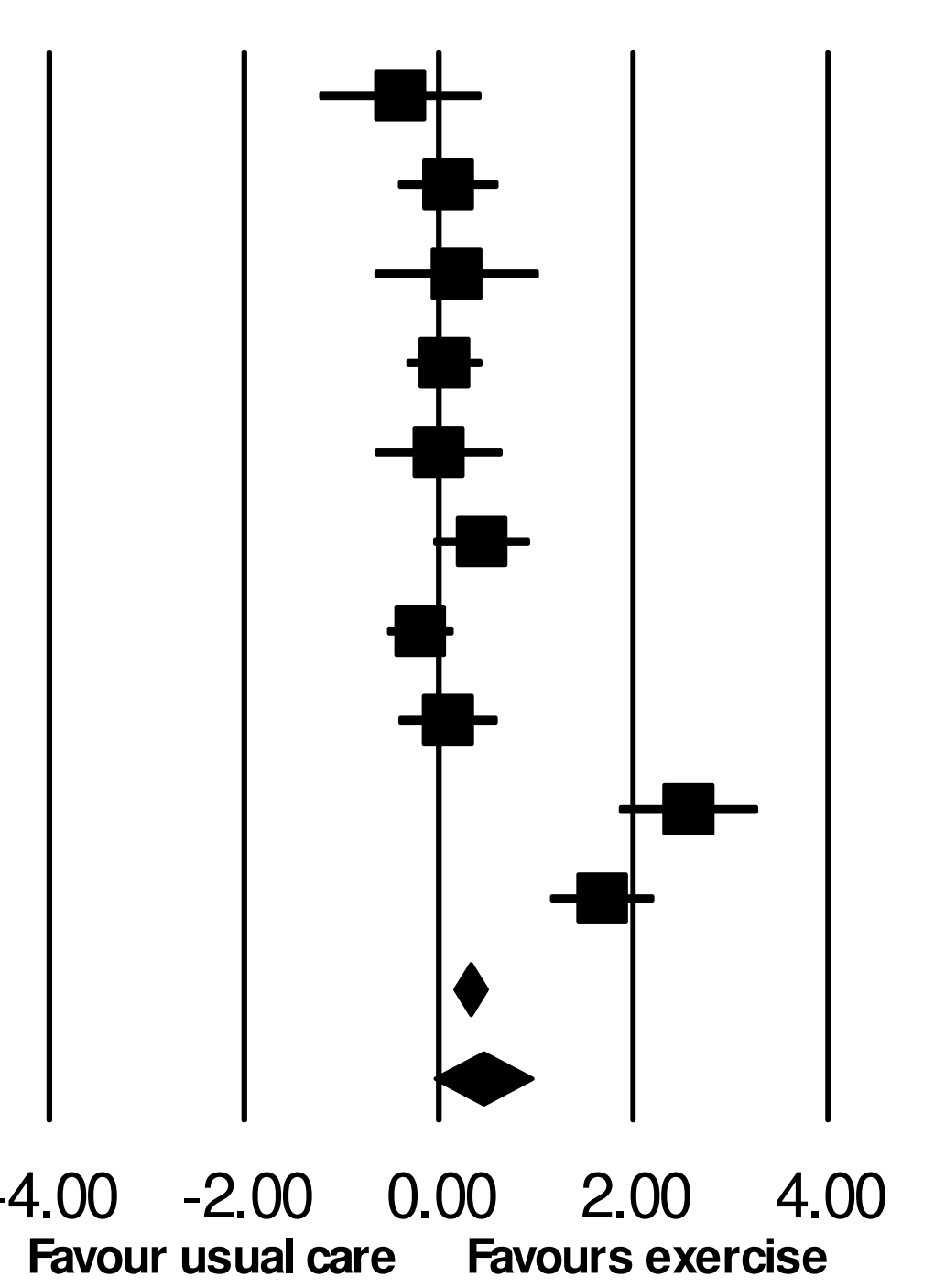

Hedges's $\mathrm{g}$ and $95 \% \mathrm{Cl}$

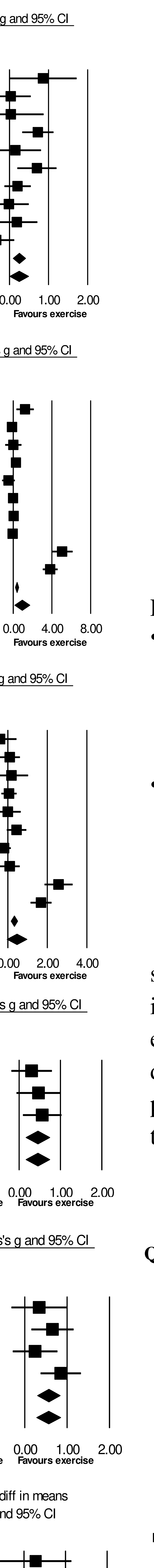

$\begin{array}{llll}-2.00 & -1.00 & 0.00 & 1.00 \\ \text { Favour usual care } & 0.00 & 0 \\ \text { Favours exercise } & 2.00\end{array}$

Hedges's $\mathrm{g}$ and $95 \% \mathrm{Cl}$

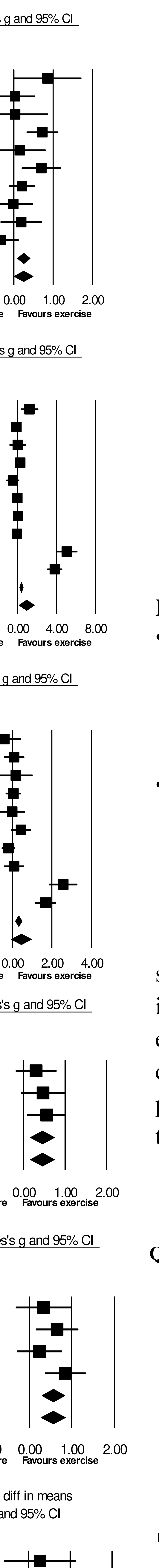

$\begin{array}{cccc}-2.00 & -1.00 & 0.00 & 1.00 \\ \text { Favour usual care } & \text { Favours exercise } & 2.00\end{array}$

Std diff in means and $95 \% \mathrm{Cl}$

\section{Background}

Prostate cancer patients, even though completed treatments, have to face risk of other treatment-related physical and psychological morbidities. Exercise has evidence to benefit physical function. However, effects of exercise on psychosocial and sexual well-being were remain inconsistent.

\section{Purpose}

The purpose of this study was to evaluate the effect of exercise interventions on physical, social, mental, and sexual function, quality of life (QOL), and depression.

\section{Method}

Relevant papers were searched in Embase, Medline, PubMed, PsycINFO, the Chinese database Airti Library, and hand searching from 1987 to 2018. The pooled means of physical, social, mental, and sexual function, sexual activity, QOL, and depression were used Comprehensive Meta-Analysis (CMA) to calculate.

\section{Results}

Pooling data from 10 randomized controlled trials (RCTs).

- Results showed that exercise intervention had significant positive effects on physical function ( $\mathrm{g}=.91,95 \% \mathrm{CI}=.14$ to 1.68$)$, sexual activity $(\mathrm{g}=.45,95 \% \mathrm{CI}=.16$ to .73$)$, sexual function $(\mathrm{g}=.56,95 \% \mathrm{CI}=.28$ to .84$)$, and social function $(\mathrm{g}=.24,95 \%$ $\mathrm{CI}=.01$ to .48$)$ in men with prostate cancer in this meta-analysis. - Only one adverse event in exercise group with higher exercise intensity at 50 to $75 \% \mathrm{VO} 2 \mathrm{max}$ ( $\geqq 70 \%$ maximum heart rate), and the participants fully recovered after.

\section{Discussion and suggestion}

Exercise can significantly improve social, physical, and sexual function. Exercise combining with psychological intervention can be as a support care for prostate cancer patients to enhance physical capacity and mental well-being. Further studies design safe and appropriated exercise programs for these cancer patients should request integrate assessment and supervision through the entire exercise program.

\begin{tabular}{|c|c|c|c|c|c|c|c|}
\hline \multirow[t]{2}{*}{ Study name } & \multicolumn{7}{|c|}{ Statistics for each study } \\
\hline & $\begin{array}{l}\text { Hedges's } \\
\text { g }\end{array}$ & $\begin{array}{l}\text { Standard } \\
\text { error }\end{array}$ & Variance & $\begin{array}{l}\text { Lower } \\
\text { limit }\end{array}$ & $\begin{array}{l}\text { Upper } \\
\text { limit }\end{array}$ & Z-Value & Value \\
\hline Monga et al. (2007) & 0.833 & 0.439 & 0.192 & -0.026 & 1.693 & 1.900 & 0.057 \\
\hline Galvao et al. (2010) & 0.288 & 0.263 & 0.069 & -0.227 & 0.802 & 1.095 & 0.274 \\
\hline Galvao et al. (2014) & 0.241 & 0.199 & 0.040 & -0.149 & 0.631 & 1.210 & 0.226 \\
\hline Jones et al. (2014) & -0.053 & 0.334 & 0.111 & -0.707 & 0.601 & -0.159 & 0.874 \\
\hline Livingston et al. (2015) & 0.122 & 0.172 & 0.030 & -0.215 & 0.459 & 0.710 & 0.478 \\
\hline Nilsen et al. (2015) & -0.472 & 0.263 & 0.069 & -0.988 & 0.043 & -1.797 & 0.072 \\
\hline Hojan et al. (2016) & 0.043 & 0.268 & 0.072 & -0.483 & 0.569 & 0.160 & 0.873 \\
\hline Hojan et al. (2017) & 3.421 & 0.368 & 0.136 & 2.699 & 4.142 & 9.288 & 0.000 \\
\hline & 0.302 & 0.090 & 0.008 & 0.125 & 0.478 & 3.349 & 0.001 \\
\hline & 0.521 & 0.325 & 0.106 & -0.117 & 1.159 & 1.600 & 0.110 \\
\hline
\end{tabular}

Figure 2. Forest plots of exercise intervention overall effects on QOL. Quality of life Model Study name

Hedges's $\mathrm{g}$ and $95 \% \mathrm{Cl}$

Heterogeneity: Chi $^{2}=85.11, \mathrm{df}=7(\mathrm{P}<0.01) ;\left.\right|^{2}=91.78 \%$ 